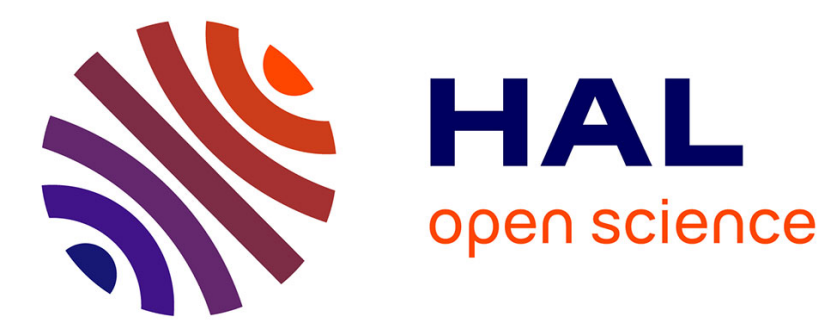

\title{
L'impossible institutionnalisation de la propriété scientifique, 1919-1939
}

Gabriel Galvez-Behar

\section{To cite this version:}

Gabriel Galvez-Behar. L'impossible institutionnalisation de la propriété scientifique, 1919-1939. L'échelle des régulations politiques, XVIIIe-XXIe siècles: L'histoire et les sciences sociales aux prises avec les normes, les acteurs et les institutions, 2019, 10.4000/books.septentrion.35497. halshs02077645

\section{HAL Id: halshs-02077645 https://shs.hal.science/halshs-02077645}

Submitted on 23 Mar 2019

HAL is a multi-disciplinary open access archive for the deposit and dissemination of scientific research documents, whether they are published or not. The documents may come from teaching and research institutions in France or abroad, or from public or private research centers.
L'archive ouverte pluridisciplinaire HAL, est destinée au dépôt et à la diffusion de documents scientifiques de niveau recherche, publiés ou non, émanant des établissements d'enseignement et de recherche français ou étrangers, des laboratoires publics ou privés. 


\section{L'IMPOSSIBLE INSTITUTIONNALISATION DE LA PROPRIÉTÉ SCIENTIFIQUE (1919-1939)}

\section{Gabriel Galvez-Behar}

Attention : ce document constitue la version d'auteur d'un
chapitre publié : GALVEZ-BEHAR, Gabriel. L'impossible
institutionnalisation de la propriété scientifique, 1919-1939 In :
L'échelle des régulations politiques, XVIII $-X X I^{e}$ siècles :
L'histoire et les sciences sociales aux prises avec les normes, les
acteurs et les institutions [en ligne]. Villeneuve d'Ascq :
Presses universitaires du Septentrion, 2019 (généré le 23 mars
2019 ). Internet :
<http://books.openedition.org/septentrion/35497>. ISBN :
9782757425145. DOI : 10.4000/books.septentrion.35497.

L'adoption du Bayh-Dole Act en 1980 aux États-Unis est bien souvent considéré comme une rupture dans le rapport que les institutions scientifiques entretiennent avec la propriété intellectuelle et, au-delà, avec la sphère économique. Rares sont les travaux, cependant, qui replacent cette loi dans un contexte historique plus large que celui du « tournant néo-libéral » et la période qui précède la Seconde guerre mondiale reste encore mal connue ${ }^{1}$. L'entre-deux-guerres constitue pourtant un moment important, caractérisé par l'extension du domaine de la propriété intellectuelle et par l'apparition, aux États-Unis, des premières politiques des universités en la matière ${ }^{2}$. Par ailleurs, au lendemain de la Première Guerre mondiale émergent de nombreux débats sur la «propriété scientifique » qui n'ont pas retenu toute l'attention qu'ils méritent ${ }^{3}$.

1. Cassier M, « L'engagement des chercheurs vis-à-vis de l'industrie et du marché : normes et pratiques de recherches dans les biotechnologies », in Alter N. Les logiques de l'innovation, Paris, La Découverte, 2202, p. 155-182 ; Mowery D.C. et alii, Ivory tower and industrial innovation : university-industry technology transfer before and after the Bayh-Dole Act in the United States, Standford, Standford University Press, 2004.

2. Robbins J., Solving the patent problem : Cognition, communication, and the National Academy of Sciences in the evolution of university patent policy, 1917-1966, PhD, université de Pennsylvanie, 2004.

3. Miller D.P., « Intellectual property and narratives of discovery invention : The league of nations' draft convention on "scientific property"and its fate », History of Science, 46, p. 299-342 ; Boudia S., « La propriété intellectuelle en science et la communauté scientifique française (1900-1930)», communication présentée au séminaire «L'invention dans les sciences et les techniques », CRHST, Paris, 6 mars 2001, En ligne $<$ http ://emlab. berkeley. edu/users/bhhall/ipconf/boudia_abs. doc $>$ [consulté le 29 novembre 2014]. 
L'objet de cette communication est de revenir sur ces débats en esquissant le jeu d'échelles qui se déploie en France et dans les arènes internationales que constituent plusieurs organismes internationaux, à commencer par la Société des nations, le Bureau international du travail, la Chambre de commerce internationale. Il s'agit notamment de décrire le rôle de certains savants français comme passeurs de ces débats entre ces différents lieux.

Nous revenons dans un premier temps sur les origines de ce débat pour, ensuite, décrire l'action de ces passeurs tant en France que dans les instances de la Société des nations. Enfin, nous nous interrogeons sur l'impact de cette controverse.

\section{LE LANCEMENT DU DÉBAT SUR LA PROPRIÉTÉ SCIENTIFIQUE (1919-1923)}

Le débat sur la propriété scientifique émerge dans le contexte d'une profonde volonté de réforme de la propriété intellectuelle en France au sortir de la Première Guerre mondiale.

\subsection{Travail intellectuel et propriété scientifique}

Il rejoint ainsi des discussions au sujet du droit d'auteur apparues depuis le début $\mathrm{du} \mathrm{XX}^{\mathrm{e}}$ siècle $^{4}$. À cet égard, le rôle du journaliste et critique Lucien Klotz, qui convoque la première réunion consacrée à la propriété scientifique, est fondamental. Lucien Klotz se fait connaître en France par son action en faveur d'une réforme du droit d'auteur. En mai 1912, dans un article de la Revue artistique, il insistait sur la dimension collective des ouvres d'art et sur la reconnaissance du travail des collaborateurs du maître :

« Il faudrait donc que la part de collaboration de ceux qui aident le génie de certains sculpteurs à se manifester, fût connue du public, que leur signature figurât au-dessous de leur illustre collaborateur et qu'on ne laissât pas clans l'ombre leur nom $»^{5}$

Sensible au caractère collectif de la création, Lucien Klotz l'est également au devenir économique des ouvres. Il est, en France, l'un des artisans de la campagne sur le droit de suite, qui aboutit à l'adoption de la loi du 20 mai 1920, conférant aux artistes un droit inaliénable sur le produit de la vente publique de leurs ouvres ${ }^{6}$. Or ce dispositif juridique inspire les premières réflexions sur la propriété scientifique, qui sont

4. Löhr I., Die Globalisierung geistiger Eigentumsrechte. Neue Strukturen internationaler Zusammenarbeit 1886-1952, Göttingen, Vandenhoeck et Ruprecht, 2010.

5. Le Droit d'auteur, 15 décembre 1912, p. 173.

6. Le Droit d'auteur, 15 février 1920, p. 15 ; Le Droit d'auteur, 15 juin 1920, p. 61 ; Duchemin J.-L., Le droit de suite des artistes, Paris, Sirey, 1948, p. 39 ; 
également nourries par toute une réflexion sur la réforme du patent system français $^{7}$.

La question du rapport des institutions scientifiques à la propriété intellectuelle est une question de longue durée ${ }^{8}$. La naissance du débat sur la propriété scientifique en France est, en revanche, étroitement liée à la question de la reconnaissance du travail intellectuel et de la représentation de ses acteurs. Il est à cet égard tout à fait notable que la question de la propriété scientifique soit posée par plusieurs organisations, syndicales ou non, chargées de représenter les cadres ou les travailleurs intellectuels. Ainsi le Syndicat professionnel des ingénieurs électriciens français l'aborde-t-il dès l'été $1921^{9}$. La Confédération des travailleurs intellectuels (CTI) s'en saisit, quant à elle, en octobre 1921. Une forme d'alliance s'établit entre ces organisations et certains savants investis dans la mobilisation scientifique durant la Grande guerre. À la suite d'une réunion tenue le 29 novembre 1921 sous l'égide du mathématicien Paul Appell, président durant la guerre de la Commission supérieure aux inventions, le Syndicat des inventeurs français « estime qu'un droit d'auteur à définir doit être accordé aux savants pour leurs découvertes utilisées dans l'industrie $»^{10}$.

Ce mouvement aboutit à l'émergence de plusieurs projets visant à reconnaître la propriété scientifique. L'un d'eux est celui de l'Union des syndicats d'ingénieurs français (USIF) qui inspire à son tour celui de Jacques Barthélémy, député et professeur-adjoint à la faculté de droit de Paris, qui dépose le 4 avril 1922 un proposition de loi sur la propriété scientifique et la réforme de la loi du 5 juillet 1844. Par ailleurs, la CTI élabore en mars 1922 un autre projet et le transmet à la Chambre des députés et à la Société des nations ${ }^{11}$. La propriété scientifique apparaît donc dans la France des lendemains de la Grande Guerre comme un problème social, qui renvoie au statut du travail intellectuel et qui mobilise des mouvements syndicaux, politiques et scientifiques.

\subsection{Propriété scientifique et coopération intellectuelle}

La fin du XIX siècle avait vu se développer les institutions internationales dans le domaine scientifique et, plus largement,

7. Galvez-Behar G. La République des inventeurs. Propriété et organisation de l'innovation en France (1791-1922), Rennes, Presses universitaires de Rennes, 2008.

8. Galvez-Behar G., «The Propertisation of Science: Suggestions for an Historical Investigation », in Isabella Löhr, Hannes Siegrist (eds.), «Intellectual Property Rights and Globalization », Special Issue, Comparativ. Zeitschrift für Globalgeschichte und vergleichende Gesellschaftsforschung, vol. 21, $\mathrm{n}^{\circ}$ 2, 2011, p. 80-97.

9. Bulletin du Syndicat professionnel des ingénieurs électriciens français, $\mathrm{n}^{\circ} 16$,

10. La Propriété industrielle, 30 juin 1922, p. 83.

11. Dalimier R. et Gallié L., La propriété scientifique. Le projet de la CTI. Création d'un droit d'auteur pour le savant et l'inventeur, Paris, Arthur Rousseau, 1923. 
intellectuel $^{12}$. Au lendemain de la Première Guerre mondiale, la mise en place de nouvelles structures de coopération intellectuelle donne lieu à des frictions entre des projets concurrents ${ }^{13}$. Les tensions entre l'Union des associations internationales, créée en 1910, et les promoteurs d'un projet largement soutenu par la France explique qu'il faille attendre la deuxième Assemblée de la SDN, en septembre 1921, pour que soit créée la Commission internationale de la coopération intellectuelle (CICI), organe de la Société destiné à promouvoir « la protection des travailleurs intellectuels, [...] à assurer le progrès des travailleurs intellectuels [et] à développer l'esprit international et la conscience de la fraternité humaine ». En mai 1922, le conseil de la SDN nomme 11 des 12 membres de la CICI, parmi lesquels Henri Bergson et Marie Curie, tous deux proches de Borel et de Langevin.

Les savants qui soutiennent le développement de la propriété scientifique en France vont porter cette question au sein de ces nouvelles organisations internationales. Lors de la première session de la CICI en août 1922, Bergson déclare :

« Il semble qu'il y ait une très grande injustice à ce que l'inventeur d'une application retire parfois des bénéfices énormes de son invention, alors que le savant qui a rendu cette invention possible n'a aucune part à ces bénéfices.

La Confédération française des travailleurs intellectuels a élaboré un projet de garantie qui na pas encore, il est vrai, un caractère international. La Commission pourrait instituer une sous-commission chargée d'examiner ce projet et d'étudier en général la question de la propriété artistique, scientifique et littéraire. $»^{14}$

Il semble bien que ce soit à la demande de la CTI que Bergson ait saisi la CICI. Le débat français prend alors une dimension internationale nouvelle d'autant que les autres membres de la Commission s'avèrent tout à fait réceptifs. Une sous-commission - composée du juriste et homme politique belge Jules Destrée, du physicien américain R. A.

12. Pham-Ti-Tu, La coopération intellectuelle sous la SDN, Genève, Droz, 1962 ; Renoliet J.-J., L'Unesco oubliée. La Société des nations et la coopération intellectuelle (1919-1946), Paris, Publications de la Sorbonne, 1999 ; Laqua D., «Internationalisme ou affirmation de la nation? La coopération intellectuelle transnationale dans l'entredeux-guerres », Critique Internationale, $\mathrm{n}^{\circ}$ 52, juillet-septembre 2011, p. 51-67 ; Kott S., « Les organisations internationales, terrains d'étude de la globalisation. Jalons pour une approche socio-historique », Critique Internationale, $\mathrm{n}^{\circ}$ 52, juillet-septembre 2011, p. 9-16.

13. Schroeder-Gudehus, Brigitte, Les scientifiques et la paix. La communauté scientifique internationale au cours des années vingt. Les Presses de l'Université de Montréal, Montréal, 1978; Rasmussen, Anne, « Réparer, Réconcilier, Oublier : Enjeux et Mythes de La Démobilisation Scientifique, 1918-1925», Histoire@Politique. Politique, culture, société, $2007<$ http ://www. histoire-politique. fr/index. php ? numero $=03 \& \mathrm{rub}=$ dossier\&item $=29>$ [consulté le $1^{\mathrm{er}}$ décembre 2014].

14. SDN - Commission de coopération intellectuelle, Procès-verbaux de la première session, Genève $1^{\text {er }}-5$ août 1922, Genève, 1922, p. 32 [neuvième séance, 5 août 1922]. 
Millikan, du juriste et sénateur italien Francesco Ruffini et du physicien espagnol Leonardo Torres Quevedo - se voit confier le soin d'établir un rapport.

Entre-temps les débats sur la propriété scientifique se développent en France. La proposition Barthélémy et le projet de la CTI donnent lieu à des réactions contrastées. Aux réactions positives s'opposent des critiques acerbes des milieux économiques et juridiques français ${ }^{15}$. Ainsi, à propos de la réunion du 29 novembre 1921, l'avocat spécialiste de propriété industrielle Fernand-Jacq déclare dans les colonnes de l'organe des économistes libéraux français, Le Journal des économistes :

« Il nous serait cruel de rappeler [...] toutes les absurdités proclamées par les braves gens que nous écoutions avec sympathie. [...] Ce qui vient d'être exposé a montré, ce me semble, le péril de lancer les savants dans l'élaboration des lois. Ils l'ont d'ailleurs bien compris eux-mêmes et se contentent de réclamer ce que tout le monde leur accorde : les moyens de travailler, de chercher, de découvrir, c'est-à-dire qu'on leur fournisse des laboratoires organisés, confortables, et des traitements suffisants.

C'est là, en réalité tout leur désir, le reste n'est que chimère ! » ${ }^{16}$

Une telle critique n'empêche pas la CTI de continuer à porter ses revendications. En janvier 1923, la première semaine des travailleurs intellectuels de France lui donne l'occasion d'intervenir en faveur de la propriété scientifique en formant le vœu de voir reconnue la propriété intellectuelle « sous toutes ses formes» « ainsi que le droit moral qui en découle $»^{17}$. Par voie de conséquence, elle considère que la propriété scientifique doit être protégée. Encore une fois, la question de la propriété scientifique est reliée à celle du droit d'auteur et, plus particulièrement, à celle du droit moral, que plusieurs juristes tentent de faire reconnaître dans la loi, voire à celle du domaine public payant ${ }^{18}$.

Tous ces débats prennent un tour nouveau avec l'adoption en août 1923 du rapport présenté par Francesco Ruffini à la CICI. Ce rapport plaide pour la reconnaissance d'une propriété intellectuelle se rapportant spécifiquement à la découverte scientifique, distincte de la propriété industrielle et de la propriété littéraire et artistique. Pour Ruffini, la propriété scientifique doit permettre une rétribution de la contribution que le savant apporte au progrès économique et dont profitent essentiellement les industriels. Pour avancer sur cette voie, il propose la constitution d'une Union internationale, sur la base des Unions existantes en matière de propriété industrielle (Union de Paris) ou de droit d'auteur (Union de Berne). Avec le rapport Ruffini, le débat prend

15. La Propriété industrielle, 31 août 1923, p. 113-118.

16. Fernand-Jacq, " Le droit de suite des inventeurs sur leurs découvertes », Journal des économistes, mars 1922, p. 332-368.

17. Informations sociales - Bureau international du travail, 16 février 1923, p. 20.

18. Bulletin de la Société d'études législatives, 1923, p. 173-219. 
alors une tournure résolument internationale.

\section{PARIS - GenÈVe - PARIS (1923-1927)}

\subsection{La quête d'un accord international}

Présenté à l'Assemblée générale de la SDN, le rapport est renvoyé pour avis aux différents pays membres. Seuls une trentaine d'États répondent et seuls dix se prononcent favorablement. Si, en France, le gouvernement fait preuve de bienveillance à l'égard des recommandations de Ruffini, les consultations lancées par le ministre de l'Instruction publique au cours de l'année 1923 donnent lieu à des réponses contrastées. L'Académie des sciences, saisie par le gouvernement, insiste sur les difficultés de la proposition de la CICI mais invite à poursuivre les efforts en faveur de cette «noble cause $»^{19}$. S'il n'est pas possible de revenir sur l'ensemble des discussions qui ont lieu en Europe, on peut toutefois indiquer la série d'articles que lui consacre l'organe mensuel du Bureau international pour la protection de la propriété industrielle ${ }^{20}$

Parallèlement d'autres projets apparaissent. Celui de Leonardo Torres Quevedo propose la création d'un fonds financé à l'aide d'une taxe sur l'industrie pour récompenser les savants ; Gariel, sous-directeur du Bureau de l'Union de Paris, reprend cette idée. Les travaux se poursuivent durant l'année 1924 au sein de la sous-commission de la propriété intellectuelle de la CICI qui propose de convoquer une commission d'experts pour étudier les différents problèmes soulevés par la propriété scientifique ${ }^{21}$. Pourtant, au cours de l'année 1924-1925, la question peine à avancer. En juillet 1925, la sous-commission de la propriété intellectuelle suggère de lancer une consultation des milieux industriels afin de relancer le mouvement ${ }^{22}$. L'Assemblée de la SDN reprend la suggestion à son compte mais demande à la CICI d'associer le Comité économique de la Société à ses travaux ${ }^{23}$. Lancée par des intellectuels et des savants, la propriété scientifique devient un enjeu de plus en plus juridique et économique.

La création en 1925 de l'Institut international de coopération intellectuelle (IICI), organe d'exécution permanent de la CICI mis à la

19. Archives de l'UNESCO (Paris), fonds de l'Institut international de coopération intellectuelle (désormais IICI), IICI 535 : CICI/PI/4 session PV I [séance du 21 juillet 1924].

20. « État actuel de la question de la propriété scientifique », La Propriété industrielle, 31 août 1923, p. 113-119; 30 septembre 1923, p. 131-136 ; 31 octobre 1323, p. 146-152 ; 30 novembre 1923, p. 169-175.

21. Idem.

22. IICI 535 : CICI/PI/ $5^{\mathrm{e}}$ session PV I [séance du 22 juillet 1925].

23. Decorzant Y., La Société des Nations et la naissance d'une conception de la régulation économique internationale, Bruxelles, Peter Lang, 2011. 
disposition de la SDN par la France, accélère toutefois les choses ${ }^{24}$. Au sein de l'IICI, la section juridique va jouer un rôle majeur dans l'instruction des débats. Raymond Weiss, fils d'une des grandes figures du droit international, André Weiss, est, au sein de la section, la cheville ouvrière du dossier. À sa personne s'ajoute celle de Marcel Plaisant, avocat spécialiste de la propriété intellectuelle et député du Cher. Devenu membre de la CICI, Plaisant devient l'un des experts de premier plan sur les questions de «droits intellectuels. » Au-delà des individus, le statut de l'IICI l'amène à travailler de conserve avec d'autres institutions liées à la SDN comme le BIT ou le Comité économique. Installé à Paris, l'Institut peut aussi collaborer avec d'autres institutions internationales comme la Chambre de commerce internationale.

C'est d'ailleurs au sein des milieux économiques que les oppositions les plus fortes se manifestent. Alors que le Comité économique de la SDN conteste la méthode de l'Institut international, la Chambre de commerce internationale se fait quant à elle l'écho des organisations représentatives des milieux industriels. Le 11 janvier 1926, une réunion rassemble des membres de la CICI et plusieurs organisations représentant le monde économique : le Comité central industriel de Belgique, le Comité des Forges, l'entreprise japonaise Mitsubishi, la Chambre de commerce internationale. Cette rencontre permet de prendre la mesure des divergences, d'importantes réserves étant formulées à l'encontre de la propriété scientifique. Dans le sillage de cette réunion, la Chambre de commerce internationale lance une enquête auprès de ses comités nationaux ; les réponses oscillent entre le refus emprunt de sympathie ou la critique acide. Par ailleurs, plusieurs industriels émettent des critiques fortes à l'encontre du projet de l'IICI : Olivetti, au nom de la Confédération générale fasciste de l'industrie italienne, considère que la « réglementation des découvertes théoriques comme «propriété scientifique » produirait une situation d'incertitude et de préoccupation dans le domaine des applications industrielles qui aurait pour conséquence inévitable d'entraver les initiatives de l'industrie. $»^{25}$

L'IICI maintient cependant le cap et réunit un comité d'experts en décembre 1927 qui donne lieu à la production d'un nouveau rapport rédigé par le sénateur et juriste Marcel Plaisant qui propose également un avant-projet de convention internationale. Bien moins ambitieux que le projet Ruffini, le rapport Plaisant de 1927 fait encore l'objet de critiques. Renvoyé aux États membres de la SDN, il reçoit les avis défavorables de plus des deux tiers de la quarantaine de pays ayant pris position.

24. Renoliet J.-J., L'Unesco oubliée..., op. cit.

25. Weiss, R, Cahiers des droits intellectuels, « La Propriété Scientifique », 1929, n² 2, p. 58 [2 avril 1926]. 


\subsection{Le projet français de législation sur la propriété scientifique}

Devenu l'objet d'une controverse internationale juridico-économique, la propriété scientifique ne semble pas intéresser outre mesure les milieux scientifiques. Ainsi l'IICI constate-t-il en 1929 que « si l'on excepte, bien entendu, les savants officiellement associés, $\mathrm{M}^{\mathrm{me}}$ Curie, par exemple, à l'activité de la Commission internationale de coopération intellectuelle, il n'a pas encore été possible d'obtenir d'un seul théoricien de la science une adhésion publique à un système concret de propriété scientifique. $\|^{26}$ Les choses avancent cependant sur le terrain national, comme en Italie, par exemple. En France, les « savants officiellement associés » profitent de l'arrivée d'Édouard Herriot à la tête du ministère de l'Instruction publique en juillet 1926. Le 12 mai 1927, un comité composé de plusieurs juristes et de hauts fonctionnaires, ainsi que du directeur du Museum d'histoire naturelle et de Paul Langevin est constitué afin de préparer un avant-projet de loi relatif aux auteurs de découvertes ou inventions scientifiques. Durant l'année 1927, la commission établit un texte. Au début de l'année 1928, une commission interministérielle est créée afin de faire de ce texte un projet de $\operatorname{loi}^{27}$. Parmi les membres de la commission, les principaux acteurs de ce débat sont notamment Émile Borel, Marcel Plaisant, Jules-Louis Breton, Paul Langevin, Marie Curie ou encore Lucien Klotz.

Plusieurs membres de cette commission interministérielle vont se tourner vers l'IICI pour obtenir un appui supplémentaire dans leur démarche. Ainsi Lucien Klotz parvient-il à obtenir l'invitation puis la nomination de Raymond Weiss au sein de la commission ${ }^{28}$. Ce dernier n'hésite pas à transmettre aux membres les plus influents de la commission la documentation préparée par l'IICI. Le soutien de l'Institut est d'ailleurs sollicité par Marie Curie pour contrebalancer les visées qu'elle jugent étatistes des hauts fonctionnaires qui n'hésitent pas à défendre l'idée d'une expropriation des droits du savant en cas de nécessitéét ${ }^{29}$ Le directeur de l'IICI donne alors la consigne à Raymond Weiss de se coordonner avec Marie Curie.

\section{L'APPORT D'UN ÉCHEC}

Le début des années 1930 voit s'intensifier les rapports entre l'IICI et les savants français engagés dans la promotion de la propriété scientifique. Ainsi Raymond Weiss se tourne-t-il vers Marie Curie pour préparer une

26. Ibidem, p. 144.

27. Journal officiel de la République française, 20 mars 1928, p.

28. IICI 182.

29. IICI 182 : Note de Luchaire à Weiss sur une conversation avec Marie Curie [30 juin 1928]. 
intervention sur la propriété scientifique devant la Société française de chimie $^{30}$. Marie Curie tente, avec un certain succès, de promouvoir la question au sein de l'Académie de Médecine. Émile Borel n'hésite pas non plus à solliciter la section juridique de l'Institut pour obtenir des informations. Une convergence de fait s'établit entre l'Institut qui tente de promouvoir la propriété scientifique au niveau international et ces acteurs scientifiques qui tentent d'avancer en France. En mai 1931, une sous-commission consacrée au droit des savants et au recrutement des chercheurs est créée au sein du Comité français de coopération intellectuelle. Présidée par Langevin, elle rassemble Marie Curie, Jean Perrin, Henri Laugier, Louis Lumière et Alfred Lacroix ${ }^{31}$. Elle est le lieu de discussions intéressantes où finissent par s'affronter deux conceptions différentes de la propriété scientifique. Pour la CTI, elle est essentiellement un droit individuel propre à chaque savant. Pour Langevin, la propriété scientifique doit être le fondement d'un « droit collectif » de la science à être financée, de manière globale, par l'industrie $^{32}$. Dans cette perspective, la propriété scientifique est un levier de financement de la science.

Aucune convention internationale sur la propriété scientifique n'est adoptée durant l'entre-deux-guerres. Aucune loi ne l'est non plus en France. De ce point de vue, la mobilisation, qui se poursuit jusqu'à la veille de la Seconde Guerre mondiale, est un échec ${ }^{33}$. Pourtant, à y regarder de plus près, les choses sont plus complexes. Le débat sur la propriété scientifique permet en France de nourrir toutes les discussions sur la question du financement de la science.

Sur le plan international, l'échec doit également être relativisé au vu des résultats de la conférence de Londres de $1934^{34}$. Cette dernière donne lieu à d'importants débats sur deux sujets en lien avec la propriété scientifique: le droit moral de l'inventeur et le statut des communications scientifiques dans la déchéance pour faute de nouveauté. Si aucun accord n'est obtenu sur le second point, malgré des initiatives fortes de la France et de l'Italie, le premier point est sanctionné par l'article 4 quinquies de la convention de l'Union de Paris révisé à Londres :

30. BNF, Manuscrits, Fonds Pierre et Marie Curie, NAF 18463, fo 151-152, lettre du 15 janvier 1931.

31. La Coopération intellectuelle, 1931, p. 231.

32. IICI 182 : Commission française de coopération intellectuelle - Feuille d'information $-n^{\circ} 14$, mai 1934 .

33. Une coordination internationale des droits intellectuelles est, semble-t-il, lancée. $L a$ Coopération intellectuelle, 1935, p. 308 À l'occasion de l'Exposition universelle de Paris en 1937 la question est à nouveau évoquée.

34. Union internationale pour la protection de la propriété industrielle, Actes de la Conférence réunie à Londres du $1^{\text {er }}$ mai au 2 juin 1934, Berne, 1934. 
Pour les pays caractérisés par le first to file, cette disposition constituait un pas symbolique mais important dans la reconnaissance du travail intellectuel qui avait inspiré les partisans de la propriété scientifique.

Au-delà des questions de propriété intellectuelle, la controverse sur la propriété scientifique a permis de porter à l'échelle internationale le problème de la place de la science, de son institutionnalisation et de son financement aux lendemains de la Première Guerre mondiale. Elle a été l'une des manières d'assurer aux scientifiques - et plus précisément à une partie d'entre eux - une présence dans de nouvelles instances internationales. Jouant de leurs engagements nationaux et internationaux, ces derniers réussissent à se servir de cette question pour avancer sur d'autres points de la régulation du travail intellectuel. 